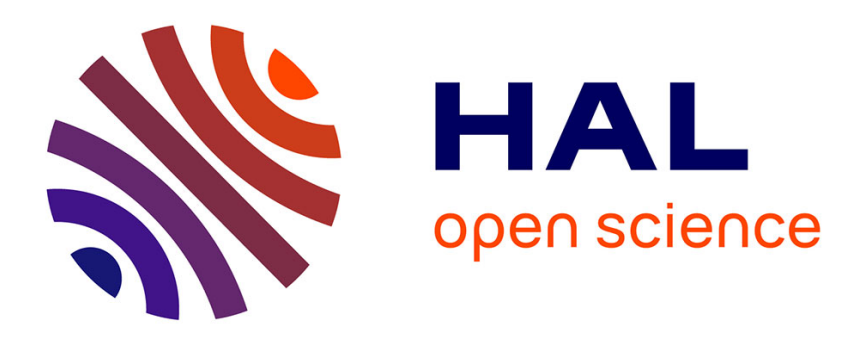

\title{
Débats intellectuels transnationaux
}

Sabrina Mervin

\section{To cite this version:}

Sabrina Mervin. Débats intellectuels transnationaux. Sabrina Mervin. Les mondes chiites et l'Iran, Karthala-IFPO, pp.301-324, 2007, 9782845868885. halshs-01858754

\section{HAL Id: halshs-01858754 https://shs.hal.science/halshs-01858754}

Submitted on 24 Aug 2018

HAL is a multi-disciplinary open access archive for the deposit and dissemination of scientific research documents, whether they are published or not. The documents may come from teaching and research institutions in France or abroad, or from public or private research centers.
L'archive ouverte pluridisciplinaire $\mathbf{H A L}$, est destinée au dépôt et à la diffusion de documents scientifiques de niveau recherche, publiés ou non, émanant des établissements d'enseignement et de recherche français ou étrangers, des laboratoires publics ou privés. 
Texte publié dans: Les mondes chiites et l'Iran, Sabrina Mervin (dir.), Karthala-IFPO, Paris, 2007, pp. 301-324.

\title{
Débats intellectuels transnationaux
}

\author{
Sabrina Mervin
}

Les débats intellectuels iraniens ont une résonance à l'extérieur du pays. Une élite chiite composée de clercs, d'intellectuels religieux, de militants, est à l'écoute de ce qui se dit, se pense et s'écrit à Qom, à Téhéran ou à Machhad. La «sphère islamique » (al-hâla al-islâmiyya) dans laquelle ils évoluent ne connaît pas de frontières, elle se recompose dès que des individus se croisent, échangent des informations et des nouvelles glanées ici et là, se remémorent des souvenirs, discutent, débattent. Des étudiants en sciences religieuses, venus de tous les coins des mondes chiites, séjournent à Qom avant de repartir porteurs d'un savoir et d'une certaine idée de la modernité islamique qu'ils vont transmettre autour d'eux. Ils peuvent se côtoyer des années pour se perdre et se retrouver au détour d'un pèlerinage, d'une visite pieuse ou de tout autre voyage, dans le majlis d'un cheikh, dans les locaux d'une revue, d'une maison d'édition ou d'un centre de recherche, dans une bibliothèque, une librairie, une fondation islamique... Les liens sont d'autant plus serrés que les hommes circulent depuis longtemps entre chiisme persan et chiisme arabe, entre l'Iran, l'Irak, le Liban et les pays du Golfe, notamment l'Arabie saoudite.

Si beaucoup sont, peu ou prou, passés par la hawza, d'autres ont étudié à l'université, et de plus en plus nombreux sont ceux qui ont suivi un double cursus. À ceux-là, il faut ajouter les autodidactes, aux enseignants, les étudiants, et aux hommes, les femmes, qui effectuent une percée. Ils ont des référents en commun, qui émanent de la culture religieuse chiite, d'un corpus de textes classiques mais aussi des textes fondateurs du mouvement islamique chiite, voire sunnite.

Ils ne partagent pas pour autant les mêmes opinions, chacun mettant ces référents à son profit pour établir sa propre position, toujours susceptible de nuances, de réexamen et de réajustements pragmatiques, au gré des événements et des nouvelles théories émises. Faut-il y voir un trait du chiisme, où l'ultime finalité des études religieuses est d'exercer l'ijtihâd, de forger son propre avis et de le défendre, et où l'individu-mujtahid prime sur l'institution? Rien n'est plus ardu que de pister des courants et des tendances afin de construire des catégories permettant d'élaborer une représentation organisée de cette sphère islamique. Acteurs et observateurs distinguent entre les islamistes et les libéraux, ou bien entre les activistes et les quiétistes, ou encore, pour reprendre le lexique propre à l'Iran, les néoconservateurs et les réformistes, les partisans du régime et leurs opposants ${ }^{1}$.

\footnotetext{
${ }^{1}$ Quiétistes, ici est à prendre dans le sens que lui a donné Nikki Keddie dans Religion and Politics in Iran. Shi'ism from Quitism to Revolution, à savoir le retrait de l'engagement
} 
Toutefois, les lignes de fracture sont parfois très ténues, car les partisans du régime islamique iranien ont aussi été marqués par la production des réformistes, ne serait-ce que pour la réfuter. Les idées de Soroush, connues de tous, même indirectement, constituent en cela le meilleur exemple puisqu'elles sont quasiment passées au sens commun dans les milieux intellectuels et religieux, en Iran et hors d'Iran. Si Soroush a été l'intellectuel religieux de la République islamique, au temps des réformistes, en prêchant pour l'individualisation de la foi, Shari'ati est devenu une référence, même au sein des mouvements islamiques, alors qu'il prônait la décléricalisation de la société. Ce ne sont pas des contradictions qui viendraient perturber un système cohérent, mais un phénomène propre à cette sphère islamique que l'on envisagera comme un champ dynamique, mouvant, parcouru de clivages subtils qui se dérobent à la catégorisation systématique. Ainsi, à Beyrouth, la «sphère islamique » chiite comprend des cercles plus ou moins proches du Hezbollah, et d'autres qui s'en détachent franchement. Chacun aura ses propres entrées en Iran, en fonction de ses positions. Celles-ci se partagent de part et d'autre de la «ligne de l'imam » (khatt al-imâm) suivie par le parti, qui repose, en grande partie, sur la théorie de wilâyat al-faqîh telle qu'elle fut conçue par Khomeini. Toutefois, là encore, des nuances s'imposent, puisque des distinctions sont établies entre la théorie et son application depuis la révolution, sachant que beaucoup caressent le rêve d'un retour à la pureté des intentions de Khomeini - qui auraient été déviées par l'exercice du pouvoir - aux fins de renouer avec l'idéal, l'utopie islamique.

\section{Une histoire commune, des référents partagés}

Les solidarités mécaniques qui lient les milieux cléricaux et, particulièrement, les élites, sont encore à l'œuvre aujourd'hui, même si l'arrivée de nouveaux venus, non issus des grandes familles dites «de science», a bouleversé le paysage. En outre, des liens organiques unissent les militants des partis et autres formations polico-religieuses. La sphère islamique est néanmoins composée d'individus aux profils disparates et aux itinéraires intellectuels singuliers qui ne sont affiliés à aucun groupe constitué. Ce qui assure sa cohésion interne et fait que chacun peut reconnaître en l'autre un interlocuteur, c'est une histoire en commun, celle du mouvement islamique chiite, qui a produit des référents auxquels tous se rattachent.

Cette histoire a des racines profondes, dans les siècles d'élaboration doctrinale $\mathrm{du}$ chiisme et la division entre usûlisme et akhbârisme, avec la victoire des premiers sur les seconds. Plus près de nous, elle a comme prémices le mouvement constitutionnaliste en Iran (1906-1911) et les débats auxquels participèrent les grands mujtahid chiites, à Qom et à

politique. Cf. Richard, Y., L'Iran, 2006, p. 258, n. 13. Sur les réformistes, voir les références citées dans la partie «L'iran des débats » de cet ouvrage et sur les néo-conservateurs, Khorokhavar, F., « Neo-conservative Intellectuals in Iran », Critique, n¹9, Fall 2001, pp. 530. 
Najaf ${ }^{2}$. C'est l'histoire du mouvement islamique chiite, qui débuta au tournant des années 1950-1960, suite à la révolution des officiers libres et du général Qâsem, en 1958, en Irak, et en réaction à la révolution blanche de Rezâ Shâh, en 1963, en Iran. Réformes agraires et mise en place de nouveaux codes de la famille affectèrent particulièrement les oulémas déjà ébranlés par la sécularisation des institutions, la baisse de leurs revenus et le lent déclin de leurs écoles religieuses. Bon nombre d'étudiants, en outre, se laissaient tenter par les attraits $\mathrm{du}$ marxisme qui se propageait dans les villes saintes : venus y apprendre les sciences religieuses, certains repartaient sans turban, et communistes ${ }^{3}$. En réponse à ces menaces, les clercs, marja' en tête, sortirent de leur position attentiste et se lancèrent dans la bataille mondaine. En Iran, ils avaient jusque là une politique d'accommodation avec le Shah ; en Irak, hormis Mahdî al-Khâlisî ${ }^{4}$, les oulémas s'étaient tus au milieu des années 1920.

Borujerdi (1875-1961) réorganisa la hozeh de Qom et tenta de consolider les institutions religieuses, tant et si bien que le nombre des étudiants y remonta ${ }^{5}$. Muhsin alHakîm (m. 1970) poursuivit les mêmes objectifs à Najaf en impulsant la création de l'association des oulémas, en 1960, qui regroupa des clercs de différentes générations et nationalités ${ }^{6}$. Toutefois, les deux grands marja ' se faisaient dépasser par de jeunes clercs plus actifs qu'eux et moins timorés face à l'engagement politique, avec lesquels ils entretinrent des relations ambiguës. À Qom, il y avait Ruhollâh Khomeini, suivi de son disciple Morteza Motahhari, et à Najaf, Muhammad Bâqir al-Sadr, entouré de quelques autres ${ }^{7}$. Ils devinrent les piliers et les idéologues du mouvement islamique chiite, dont leurs œuvres sont, aujourd'hui, les principales références.

Muhammad Bâqir al-Sadr était avant tout un faqîh. Enseignant à la hawza, il s'est employé à rénover les sciences juridiques islamiques, particulièrement les usûl, afin de les mettre à la portée des étudiants ; ses ouvrages en la matière sont aujourd'hui des manuels couramment utilisés ${ }^{8}$. En outre, il est connu pour deux livres visant à réfuter la philosophie occidentale et le marxisme qui ont introduit ces domaines dans la hawza et font aujourd'hui

\footnotetext{
${ }^{2}$ Hairi, A. H., Shi 'ism and Constitutionalism in Iran, 1977 ; Martin Martin, V., Islam and Modernism : the Iranian Revolution of 1906, 1989.

${ }^{3}$ NAEF, S., "Shî‘'î-shuyû‘'̂̀ or: How to become a Communist in a Holy City », The Twelver Shia in Modern Times, Ende, W. et Brunner, R. éd., 2001, pp. 255-267.

${ }^{4}$ Lire sa biographie, traduite par Pierre-Jean Luizard, La vie de l'ayatollah Mahdî al-Khâlisî par son fils.

${ }_{5}^{5}$ MARTIN, V., Creating an Islamic State, 2003, p. 50-56.

${ }^{6}$ A. JABAR, F., The Shi 'ite movement in Iraq, 2003. p. 110-113.

${ }^{7}$ Citons, entre autres, Muhammad Bahr al-'Ulûm, Murtadâ al-'Askarî, Muhammad Bâqir alHakîm, ensuite fondateur de l'ASRII, Muhammad al-Sadr (père de Muqtadâ), et les Libanais Muhammad Husayn Fadlallâh et Muhammad Mahdî Shams al-Dîn.

${ }^{8}$ Notamment al-Ma'âlim al-jadîda, une très claire introduction aux usûl, et Durûs fì 'ilm alusûl, plus ardu, traduit en anglais par Roy Mottahedeh. Cf. as-Sadr, Muhammad Bâqir, Lessons in Islamic Jurisprudence, 2003.
} 
référence dans les milieux islamiques, Notre philosophie, et Notre Économie ${ }^{9}$. Il n'était pas le seul à tenter de jeter les bases d'une économie islamique, capable de s'ériger en troisième voie à la fois face au socialisme et au capitalisme. Déjà, dans les années 1940, Sayyid Qutb s'était déjà penché sur la justice sociale en islam et, côté chiite, Mahmûd Tâleqâni avait étudié la question de la propriété. Toutefois, par sa connaissance de la terminologie marxiste, Muhammad Bâqir al-Sadr inaugura un nouveau rapport au matérialisme occidental, qu'il s'agissait de bien connaître pour mieux le réfuter. En cela, il influença, d'abord, ses proches et ses contemporains, à commencer par son cousin Mûsâ al-Sadr, qui écrivit des articles sur l'économie dans la revue Maktab-e eslâm, publiée à Qom, se focalisant sur la question de la distribution, amplement traitée par Muhammad Bâqir ${ }^{10}$. Notons que Mûsâ al-Sadr était alors en contact avec Muhammad Beheshti, qui, lui aussi, publia sur l'économie islamique et particulièrement sur le système bancaire ${ }^{11}$.

Au plan politique, Muhammad Bâqir al-Sadr élabora une conception originale de l'État islamique qui évolua sensiblement entre les années 1960 et la fin des années 1970. Sa théorie, différente de celle de Khomeini, prône le militantisme et l'institutionnalisation du clergé, mais sépare les pouvoirs qu'il répartit entre la nation et les clercs, tous deux redevables devant la Constitution et la loi. Aussi peut-on parler d'une « hiérocratie libérale », ou d'un système «populiste semi-libéral ${ }^{12}$. Auteur prolixe, Muhammad Bâqir al-Sadr fut aussi l'animateur d'une revue, Adawâ' (Lumières), à laquelle collaborèrent de près de jeunes clercs libanais, Muhammad Husayn Fadlallâh et Muhammad Mahdî Shams al-Dîn ${ }^{13}$. Il fut aussi un acteur du mouvement islamique, en tant qu'inspirateur du parti al-Da'wa, avec lequel ses relations restent toutefois mal élucidées. Peu après la révolution iranienne, Muhammad Bâqir al-Sadr était devenu trop dangereux aux yeux du régime baathiste qui le fit exécuter, en avril 1980 , et les membres d'al-Da'wa furent pourchassés. Ils se dispersèrent géographiquement et divergèrent dans leurs options politiques, ce qui causa la ramification du parti en plusieurs branches. Reste que l'expérience d'al-Daw'a et les idées de Muhammad Bâqir al-Sadr constituent les fondements du mouvement actuel, particulièrement pour les chiites arabes, et que tous les partis se réfèrent plus ou moins à lui.

\footnotetext{
${ }^{9}$ Falsafatunâ (1959) et Iqtisâdunâ (1961) sont régulièrement réédités en arabe, et traduits au moins partiellement par des maisons d'édition islamiques. Sur ces ouvrages, cf. Mallat. Voir aussi « Notre économie », John Donohue, J., Cahiers de l'Orient, n ${ }^{\circ}$-9, 1988, pp. 179-202.

${ }^{10}$ A. Jabar, F., p. 296-305, donne un aperçu de la théorie de Muhammad Bâqir al-Sadr sur la distribution. Voir aussi Chehabi, Distant relations, p. 148.

${ }^{11}$ Cf. Beheshti, M., al-Iqtisâd al-islâmî, 1997. Sîra wa hayât, 2000, pp. 36-37. Muhammad Beheshti, élève de Khomeini, joua ensuite un rôle majeur dans l'instauration de la république islamique. Il fut tué dans l'explosion d'une bombe au siège du parti de la république islamique, à Téhéran, en juin 1981. Digard L'Iran au XX siècle, p. 184.

${ }^{12}$ Selon les expressions utilisées par A. Jabar, F., The Shi'ite Movement, p. 281, dans un exposé sur la théorie politique de Muhammad Bâqir al-Sadr, cf. pp. 280-287. Le clerc iranien Mohsen Kadivar, dans son étude sur les théories de l'État dans le droit islamique chiite, classe celle de Sadr parmi les théories de l'État islamique électif (intikhâbî). Nazariyyât al-dawla, 2004, p. 185.

${ }^{13}$ A. Jabar, p. $114 s q$.
} 
Les doctrines de Ruhollâh Khomeini forment un autre volet de ces fondements. Elles s'inscrivent en partie dans la tradition des utopies islamiques passant par Farâbî, Ibn 'Arabî, et Mollâ Sadrâ Shirâzi, elles-mêmes inspirées par le concept du sage vertueux qui gouverne la cité dans la République de Platon. Khomeini, en effet, n'était pas seulement le spécialiste du droit islamique et l'homme politique que l'on connaît, il était aussi un gnostique et ses écrits sur le gouvernement islamique s'en ressentent ${ }^{14}$. Il enseigna la philosophie mystique ('erfân), à Qom, à partir des années 1940, alors que c'était mal vu dans les milieux cléricaux ; aujourd'hui, on le crédite de la réintroduction de cette discipline dans la hozeh. Le principal apport de Khomeini fut donc de conjuguer philosophie mystique, fiqh et vision politique. De là découlèrent ses théories du gouvernement islamique et du velâyat-e faqih, qu'il professa durant son exil à Najaf, conférant les pouvoirs de l'imam attendu au théologien-juriste (faqîh) qui s'érige ainsi comme le guide de la communauté, aussi bien aux plans spirituel que politique. Cette théorie, ensuite réajustée, allait devenir la base du régime islamique de l'Iran, mais aussi un objet de débat entre les oulémas.

Si Khomeini fut un théoricien mystique qui se transforma en politicien pragmatique quand il s'agit d'exercer le pouvoir, son disciple Motahhari fut le véritable idéologue du régime islamique qui l'a surnommé «le maître de la révolution » (mo'allem-e enqelâb) et commémore son assassinat, chaque année ${ }^{15}$. C'est lui qui élabora le système de pensée sur lequel s'appuya le régime pour encadrer, mobiliser et organiser la société. Théosophe comme son maître, Motahhari en partageait une certaine vision du monde. Néanmoins, il s'intéressait plus que lui aux questions sociales et fut un clerc réformiste avant d'être un militant révolutionnaire. Il vilipendait les clercs conservateurs, notamment pour leur frilosité à s'engager en politique, et ambitionnait de moderniser les institutions religieuses ${ }^{16}$. Dans sa volonté de changer la société pour la rendre plus juste, Motahhari se heurta à la concurrence de tous ceux qui, dans les années 1970, en Iran, étaient influencés par le léninisme et le tiersmondisme, autant dire des idées importées d'Occident, pour lui à bannir. Ainsi, il s'engagea dans la critique systématique du marxisme, non sans l'avoir étudié de près, avec les moyens dont il disposait, et dans celle du socialisme islamique de Shari'ati, qu'il combattit âprement. Tout mouvement de sécularisation était à ses yeux un complot contre l'islam ainsi que toute tentative d'intégration de concepts étrangers aux doctrines islamiques. Son idéologie, à l'instar de celle que construisit Muhammad Bâqir al-Sadr, se voulait une alternative, un rempart aux matérialismes occidentaux. Toutefois, à l'instar de ce dernier et

\footnotetext{
${ }^{14}$ Vanessa Martin, p. 31-47. Pour l'aspect gnostique des idées de Khomeini, on se référera aussi à Bonaud, C. Y. L'Imam Khomeiny, un gnostique méconnu du XXe siècle, 1997.

${ }^{15}$ NiKPEY, A, Politique et religion en Iran contemporain. 2001, p. 221. Motahhari fut assassiné en mai 1979 par un membre du groupe Forqân.

${ }_{16}$ Sur son programme de réforme de l'institution cléricale, cf. Motahhari, M., «The Fundamental problem in the Cerical Establishment», introduit par Hamid Dabashi, in Walbridge, L. (ed.) The most Learned of the Shi' $a$, p. 161-182.
} 
d'autres penseurs islamistes, il édifia lui-même un système hybride, influencé par les idées qu'il réfutait. Auteur prolifique, il a une œuvre variée qui a fait l'objet de moult rééditions ${ }^{17}$.

Au bout du compte, Muhammad Bâqir al-Sadr et Mortaza Motahhari sont souvent cités, aujourd'hui, par les acteurs de la sphère islamique comme les pères de l'idéologie islamique chiite, même si d'autres auteurs se greffèrent sur leurs idées et les développèrent ou les nuancèrent pour fonder leurs propres mouvements. Reste que la particularité des chiites est que leurs idéologues, hormis quelques « intellectuels religieux » iraniens comme Shari'ati, sont des clercs de haut rang dans la hiérarchie religieuse. Les idéologies qu'ils construisent ont pour objectif premier de rejeter le processus de sécularisation touchant les sociétés musulmanes, ainsi que l'influence occidentale, vue comme un effet de l'impérialisme. Pour se faire, ils s'efforcèrent de moderniser leurs institutions (marja 'iyya et hawza), en allant contre l'inertie des milieux cléricaux. En outre, il s'en prirent aux philosophies matérialistes auxquelles ils opposèrent le messianisme chiite et un mélange de théories rationalistes, réformistes et modernistes ou bien mystiques, revisitées à la lueur des idéaux tiers-mondistes. L'islam devait s'ériger en un système total, répondant à tous les maux de la communauté. Plus question de suivre l'attitude quiétiste, la taqiyya des anciens, mais passer à l'action, à l'activisme politique afin de ne plus attendre passivement le retour du Mahdî.

Les mots d'ordre furent tirés du Coran. Ainsi, la lutte entre le bien et le mal, l'opprimé et l'oppresseur fut décrite en des termes opposant les déshérités (mustad'afûn) ${ }^{18}$ aux arrogants de ce monde (mustakbarûn). Les masses furent mobilisés durant les célébrations religieuses, notamment celle de Achoura, par le biais de slogans qui n'ont pas perdu de leur puissance évocatrice et sont désormais brandis chaque année. Ainsi, lors ces célébrations qui suivirent la guerre de juillet 2006 contre Israël, le Hezbollah libanais proclamait «la victoire de l'opprimé » (intisâr al-mazlûm), se référant, une fois encore, au paradigme de Husayn, le martyr de Karbala. Cette fois, le parti y mettait un sens nouveau, puisqu'il ne s'agissait plus pour lui d'une victoire symbolique ou morale, mais d'une victoire réelle, celle qu'il affirmait avoir remportée.

Si les concepts de la tradition chiite firent l'objet d'une relecture idéologique, les modes d'organisation propres aux partis léninistes furent mis à profit, système pyramidal et culte du secret par exemple, afin d'assurer l'efficacité de l'action. Que ce soit par les concepts élaborés, les formes et les outils de la mobilisation, le but consistait à produire et à promouvoir une idéologie porteuse d'une modernité islamique. En fait, depuis la fin du XIXe siècle, les sociétés musulmanes - et leurs clercs - se heurtent à la modernité imposée

\footnotetext{
${ }^{17}$ Martin, V., Creating..., chap. Chap IV, « Motahhari : Towards an Islamic Ideology and the Battle for Control of Political islam»; NIKPEY, A, p. 218-230. Sur ses attaques contre Shari'ati, Rahnema, Ali, An Islamic Utopian, chap. 16 et 17.

${ }_{18}$ Mûsâ al-Sadr, au Liban, utilisa le terme mahrûm, traduit habituellement par déshérité ; il lança Harakat al-mahrûmîn, le mouvement des déshérités.
} 
par un Occident conquérant et hégémonique, et cherchent les réponses à lui présenter ${ }^{19}$. Les mouvements islamiques chiites «révolutionnaires », Iraniens en tête depuis 1979, ont apporté les leurs.

Cependant, un courant plus discret, dès avant la révolution, frayait son chemin et en proposait d'autres. Déployant une vision de l'islam plus humaniste que politique, il ne rejette totalement ni la sécularisation qui touche les sociétés musulmanes, ni les idées importées d'Europe. Deux types d'acteurs sociaux ont porté ce courant, souvent qualifié de réformiste : des clercs et des intellectuels religieux. Rappelons la difficulté de catégoriser les hommes et leurs idées, soulignée plus haut. Les clercs dont il s'agit sont devenus, de plus en plus informés sur les affaires du monde, et parmi eux, on trouve une élite versée en sciences profanes ou en théologie chrétienne. Ainsi de Mohammad Mojtahed Shabestari, qui prit la direction du centre islamique de Hambourg, fondé à l'instigation de Borujerdi, en 1969. Le «docteur ayatollah Beheshti » l'avait précédé à ce poste ${ }^{20}$, et Mohammad Khâtami lui succéda. Shabestari resta une dizaine d'années à Hambourg, où il suivit des études de philosophie et de théologie chrétienne avant de revenir à Téhéran, juste avant la révolution, sachant l'arabe, l'anglais et l'allemand ${ }^{21}$. De la même manière, les intellectuels, pour la plupart des universitaires, quelle que fût leur spécialité, se formèrent aux sciences religieuses, à l'instar de Soroush. Tant et si bien que des universitaires enseignent à la hozeh, surtout dans les écoles réformées, alors que des religieux donnent des cours à l'université, particulièrement dans les départements de philosophie (comme Shabestari et Kadivar). Enfin, certains ne portent pas le turban, même s'ils sont intégrés dans le système de la hozeh, comme Malekiân.

Si l'on retrouve aujourd'hui ce phénomène dans la hâla islâmiyya, au Liban et en Irak notamment, il y est moins courant qu'en Iran où la « révolution culturelle » (enqelâb-e farhangi) l'a systématisé. En 1980, après la fermeture des universités, Khomeini mit en place plusieurs instances chargées d'organiser une forme de rapprochement entre la hozeh et les universités, qui rassemblaient des intellectuels et des religieux. Après des mois de discussions, ayant introduit quelques matières «islamisées » dans les programmes, certains universitaires participant au projet estimèrent qu'ils ne sauraient aller plus loin dans ce processus de réforme ${ }^{22}$. À l'opposé, d'autres, notamment des clercs, voulaient procéder à une complète islamisation des sciences sociales et établir un système de passerelles durable entre

\footnotetext{
${ }^{19}$ Les représentations des médias taxant le régime iranien de « rétrograde » ou « médiéval », ont fait oublier qu'il résulte d'une rupture avec les doctrines classiques et d'une tentative de construire une modernité endogène Sur ce point, cf. Juan Cole, Sacred spaces, "The Modernity of Theocracy », pp. 189-210.

${ }^{20}$ Dans les années 1960, Beheshti qui savait l'allemand et l'anglais, faisait figure d'exception, la grande majorité des clercs n'avaient pas terminé leurs études secondaires. Richard, Y., « Le rôle du clergé : tendances contradictoires du chi 'isme iranien contemporain ", Archives des sciences sociales des religions, 55/1, 1983, p. 14. Notons que son compagnon Mûsâ Sadr, qui s'illustra au Liban, avait lui aussi suivi un double cursus.

${ }^{21}$ Amirpur, K., « Mohammad Mojtahed Shabestari », Orient, 2000 ; Wâsifî, al-Fikr al-islâmî al-mu'âsir fî Irân.p. 315.

${ }^{22}$ Entretien avec Gholamabbas Tavasoli, université de Téhéran, 7/4/04.
} 
l'université et la hozeh; ils poursuivirent leurs efforts en ce sens, avec l'appui du régime. Ainsi, du cheikh Mohammed Taqi Mesbâh Yazdi, qui, dans le cadre du comité Hozeh o daneshgâh (hozeh et université), avait organisé des échanges et patronné l'initiation d'une centaine d'universitaires aux sciences islamiques, avec l'objectif de les mener à critiquer les sciences sociales à la lumière de l'islam. De retour à l'université, ceux-ci devaient se faire les piliers de la révolution culturelle. Si ce projet ne s'est pas réalisé, le cheikh radical a continué d'œuvrer pour la coopération entre les deux institutions et pour l'adoption d'un point de vue islamique sur les sciences sociales, tant dans ses écrits qu'au sein de l'institution d'enseignement qu'il dirige, à Qom ${ }^{23}$. Quant au centre Hozeh o daneshgâh, ses activités ont pris plus d'ampleur depuis une dizaine d'années, particulièrement l'édition d'ouvrages visant à islamiser les sciences sociales ${ }^{24}$.

L'expérience iranienne en la matière est souvent érigée en modèle, au Liban, par exemple, où les milieux de la hozeh souhaiteraient établir des ponts avec l'université afin d'obtenir des équivalences pour leurs étudiants. Toutefois, cette expérience est aussi critiquée, tant à l'intérieur qu'à l'extérieur de l'Iran, non seulement par les intellectuels mais aussi par des clercs réformistes. Schématiquement, encore une fois, il s'agit se savoir si l'on veut islamiser la modernité ou moderniser l'islam. Les tenants de la seconde option penchent pour le recours aux sciences sociales, acceptées comme universelles, afin de développer leur compréhension des doctrines.

\section{Une nouvelle théologie}

Dans les milieux réformistes, en persan comme en arabe, on parle de nouvelle théologie, ou théologie moderne ('ilm al-kalâm al-jadîd), en la distinguant de l'ancienne science du kalâm, tant par ses méthodes que par son objet. Celui-ci s'est étendu à de nouvelles questions qui n'étaient pas prises en compte dans le kalâm classique, et les outils mis en œuvre pour y parvenir dépassent les sciences islamiques pour s'élargir aux sciences humaines telles qu'on les pratique aujourd'hui. En outre, cette nouvelle théologie ne vise plus à défendre la religion, mais à la comprendre. En Iran, le clerc qui la représente le mieux, et qui est devenu la référence en la matière, est Mohammad Mojtahed Shabestari. S'il a fait des émules, il a aussi ses détracteurs mais, à l'instar de Soroush, fait désormais partie du paysage cognitif et ne peut être ignoré de qui s'intéresse au sujet. Il a relativement peu produit et se concentre désormais sur l'enseignement et ses recherches pour une encyclopédie islamique ${ }^{25}$, mais il a marqué toute une génération. En outre, son statut de clerc et son éducation à la hozeh

${ }^{23}$ Entretien avec Mohammed Taqî Mesbâh Yazdi, Qom, 19/8/03. Une illustration de sa méthode peut être trouvée dans son ouvrage Usûl al-ma'ârif al-insâniyya, qui regroupe des conférences qu'il a données a Beyrouth, en arabe.

${ }^{24}$ Entretien avec le sayyed 'Arabi, Qom, 1/1/2004. On trouvera des informations sur le site de cette institution, devenue centre de recherche affilié à la hozeh de Qom, en 1999 sur: http://www.hawzeh.ac.ir.

${ }^{25}$ Dâ'erat-e bozorg-e eslâmi, à Téhéran. 
de Qom, où il a étudié de 1950 à 1968, lui confèrent plus de crédibilité dans les milieux religieux. Proche de Beheshti, il a aussi fait partie du comité éditorial de la revue Maktab-e eslâm, qui traitait de questions sociales et politiques. Shabestari jouit par ailleurs de solides connaissances en matière de théologie catholique et protestante, acquises lors de son séjour à Hambourg, pendant dix ans. Il est à la fois mujtahid et docteur en philosophie et ses influences s'en ressentent.

Shabestari s'appuie sur les écrits de «'Allâma » Tabatabâ'i (m. 1981). C'est dire qu'il se réfère à la tradition philosophique islamique dans ses manifestations les plus contemporaines. Célèbre exégète, Tabâtabâ'i fut aussi un vulgarisateur de la pensée de Mollâ Sadrâ Shirâzi, qu'il introduisit dans la hozeh en y enseignant le Livre des quatre voyages ${ }^{26}$. Or, pour Shabestari le voyage spirituel ne mène pas à l'annihilation en Dieu, comme c'était le cas pour les idéologues Motahhari, Khomeini, ou même Shari'ati, mais vers la conscience de soi et l'affirmation de l'homme et de sa liberté. Il a donc une autre approche du 'irfân, qu'il n'associe pas à l'islam comme un système total susceptible de répondre à tous les problèmes posés par la modernité ${ }^{27}$. Bien plus, si Shabestari reprend les hypothèses métaphysiques du maître, il n'en constate pas moins l'incapacité de la philosophie classique, comme celle du fiqh, à renouveler sa compréhension du monde. Le recours aux sciences modernes est donc nécessaire pour fonder les sciences qui manquent à l'islam, comme la philosophie du droit, la philosophie morale, politique, et économique ${ }^{28}$.

L'apport de Shabestari est d'avoir démontré l'aspect limité et changeant du savoir religieux, alors qu'il est déclaré éternel - en cela il rejoint Soroush. Aux penseurs, donc, de s'adapter au siècle et de développer une démarche et un appareil critique adéquats. Ce qu'il fit lui-même pour poser son herméneutique du Coran et de la sunna (1996), qui constitue l'une de ses œuvres majeures, avec son ouvrage sur la foi et la liberté, et sa Critique de la lecture officielle de la religion, publié en 2000. Mojtahed Shabestari a par ailleurs participé au débat public en Iran, en écrivant des articles dans les revues.

Cependant, la pensée de Shabestari et d'autres auteurs iraniens dépassait les frontières. Dès 1995, la revue al-Muntalaq publia un entretien avec lui, où après avoir expliqué que cette nouvelle théologie en était encore à ses balbutiements, il se félicitait de l'existence, déjà, d'un «noyau », et souhaitait voir se succéder les contributions et les débats ${ }^{29}$. Al-Muntalaq est la revue-type de la sphère islamique libanaise, dont l'histoire s'est écrite en partie dans ses pages, au fil des années. Elle fut créée à Beyrouth en septembre 1977 par l'Union libanaise des étudiants musulmans, organisation patronnée par Muhammad Husayn Fadlallâh, qui en fit

\footnotetext{
${ }^{26}$ Cf. Dabashi, H.,Theology of Discontent, chap. 5 et Ridgeon, L., Religion and Politics in Modern Iran, chap. 8.

${ }^{27}$ Vahdat, F.« Post-revolutionary Discourse... part I », 2000, pp. 41-43.

${ }^{28}$ Id. p. 39 et Sadri, M. « Sacral Defense of Secularism... », 2001, p. 261.

${ }^{29}$ Jâbir, H., «al-Tajdîd wa al-taqlîd fî̀ al-fikr al-îrânî al-mu'âsir », Shu'ûn al-sharq al-awsat, 103, été 2001, p. 46.
} 
sa tribune, dans les années $1980{ }^{30}$. Elle se fit ensuite l'écho des discussions menées au sein du mouvement islamique chiite au Liban et, à partir de la seconde moitié des années 1990, Hasan Jâbir, son rédacteur en chef, s'employa à faire connaître les penseurs iraniens. En 1998, il publia un dossier sur «le renouveau de la théologie », contenant quatre articles traduits du persan vers l'arabe, présentant des visions différenciés de la question ${ }^{31}$. Pendant quelques années, ce fut une sorte d'euphorie, cette nouvelle approche ouvrait des champs qui n'étaient pas pris en compte par le chiisme classique et permettait de poser des questions émanant de la réalité, de l'expérience. L'expérience en question, c'était aussi celle du gouvernement islamique, en Iran et de ses limites. «Avant, on réfléchissait sur les relations entre Dieu et son serviteur, maintenant, on envisage les problèmes de l'homme dans la société, de la politique (shûrâ et démocratie), et cela implique une accommodation et un renouveau dans toutes les sciences religieuses ${ }^{32}$. Hasan Jâbir lui-même choisit de réfléchir sur la philosophie du fiqh en s'intéressant aux valeurs qui prévalent aux normes et les sous-tendent ${ }^{33}$, et il continue aujourd'hui dans cette voie. Universitaire, il se mit à enseigner la nouvelle théologie dans une hawza $a^{34}$.

L'enthousiasme pour la nouvelle théologie s'était propagé ${ }^{35}$. À Qom, il avait gagné les cercles des étudiants et des enseignants de la hozeh non iraniens, qui avaient accès aux textes en persan. Des Irakiens, des Libanais, des chiites du Golfe se retrouvaient dans des réunions informelles pour en débattre. En 1994, l'un d'eux, 'Abd al-Jabbâr Rifâ‘î, fonda une revue qui allait devenir un véritable pont entre les champ intellectuels en Iran et dans le monde arabe, bien plus, un forum transnational entre penseurs chiites et sunnites : Qadâyâ islamiyya $m u^{\prime}$ âsira (Questions islamiques contemporaines) ${ }^{36}$. La production foisonnante de 'Abd alJabbâr Rifâ‘î mérite que l'on s'arrête sur son itinéraire intellectuel. Né au sein d'une famille de paysans, dans un village des alentours de Nasriyya, il put fréquenter l'école élémentaire qui s'y ouvrit après la révolution de 1958. Ensuite, il commença un périple qui le mena à Bagdad, où il acheva un cursus d'agronomie, puis à la hawza de Najaf, où il étudia de 1978 à 1980. La répression contre les chiites l'obligea à quitter l'Irak et, en 1984, il se retrouva à Qom. Se disant toujours «en quête de science», il y enseigne aujourd'hui, à la hozeh et à l'université et, s'il ne porte pas le turban, c'est un « cheikh». Il reçut une formation classique, expérimenta toutes les possibilités offertes par la liberté de choix qui caractérise le système d'enseignement de la hozeh, avant de faire la critique de ce système, en vue « de le moderniser et de l'ouvrir au siècle ».

\footnotetext{
${ }^{30}$ Sankari, J., Fadlallah. The Making of a Radical Shi'ite Leader, 2005.

${ }^{31}$ Al-Muntalaq, $\mathrm{n}^{\circ} 119$, automne-hiver 1997-98.

${ }^{32}$ Entretien avec Hasan Jâbir, Beyrouth, 28/12/01.

${ }^{33}$ Jâbir, H., al-Maqâsid al-kulliyya wa al-ijtihâd al-mu 'âsir, 2001.

${ }^{34}$ Entretien à Beyrouth,13/4/03.

${ }^{35}$ Entretien avec 'Abd al-Jabbâr al-Rifâ‘̂̂, Qom, 28/8/03.
} 


\section{Qadâyâ... et ses sœurs}

L'entreprise consiste en une véritable reconstruction des sciences religieuses et de la représentation du divin, et c'est l'objectif de sa revue. Elle donne en outre la parole à des groupes d'étudiants et de clercs de la hozeh, qui, selon Rifâ‘î, «n'avaient pas de voix auparavant ${ }^{37}$. Seul, quasiment sans aide, 'Abd al-Jabbâr Rifâ‘î a publié, en arabe, non seulement des philosophes comme Shayegan et des penseurs réformistes comme Soroush, Shabestari, ou Malekiân, mais les auteurs qui réfutaient leurs idées. "Qadâyâ n'essaie pas d'établir une position qu'elle estime juste, explique Rifâ‘̂̂, mais d'exposer des opinions variées au lecteur... l'important est d'ouvrir des brèches pour déclencher la réflexion, ce qui ne peut se faire qu'en suscitant des questions parce que l'histoire de la pensée est celle des grandes questions soulevées ${ }^{38}$. Autant dire que les articles publiés sont parfois très contrastés, même si, comme il est d'usage dans ce type de publications, le ton employé et certaines formules édulcorent le propos. Quelques oulémas de Qom y ont écrit, tels Mohammad Taqi Mesbâh Yazdi, Ja‘far Sobhâni et Nâser Makârem Shirâzi. Des réformistes libanais, comme Muhammad Mahdî Shams al-Dîn, Muhammad Husayn Fadlallâh, Muhammad Hasan al-Amîn ou Hânî Fahs ont encouragé l'éditeur de la revue. Celle-ci est publiée à Beyrouth et diffusée dans le monde arabe, particulièrement en Égypte et au Maroc, où elle trouve un public. On y lit d'ailleurs des textes de l'égyptien Hasan Hanafî « plus porté sur l'idéologie que l'épistémologie », comme des textes d'auteurs maghrébins. Si 'Abd alJabbâr Rifâ‘î note que les penseurs maghrébins, contrairement aux iraniens, sont extérieurs à la sphère islamique, il souligne la proximité de leurs idées. Ainsi de Muhammad Talbi, 'Abd al-Majîd Charfî, Abdou Filali Ansari... sans oublier Muhammad Arkoun, selon lui le plus marquant.

Hormis les textes d'auteurs et les entretiens, que la revue publie ou traduit du persan, elle rend compte de débats et tables rondes qu'elle organise ou reprend de revues en persan, comme Naqd o nazar, qui émane de la hozeh de Qom. Son éditeur saisit toutes les occasions qui se présentent pour réunir des penseurs et consigner leurs échanges. Les thèmes des dossiers présentés par Qadâyâ islâmiyya mu'âsira depuis 1998 sont très révélateurs de l'évolution des questions qui font débat aujourd'hui. On y trouve des études sur : la pensée politique chiite $\left(\mathrm{n}^{\circ} 1\right.$ et 2,1998$)$ la pensée islamique contemporaine et ses nouvelles orientations $\left(n^{\circ} 5,1999 ; n^{\circ} 26,2004\right)$; l'exégèse coranique $\left(n^{\circ} 4,1998\right)$ avec la publication de la traduction de l'ouvrage de Shabestari sur l'herméneutique $\left(n^{\circ} 6,1999\right)$; la philosophie du droit islamique et une relecture de l'approche par les maqâsid (nº et 8, $\left.1999 ; \mathrm{n}^{\circ} 13,2000\right)$; la doctrine de Muhammad Bâqir al-Sadr ( $\mathrm{n}^{\circ} 11$ et12, 2000); la nouvelle théologie $\left(\mathrm{n}^{\circ} 14\right.$, 2001); le libéralisme ( $\left.n^{\circ} 24-25,2003\right)$; la tolérance $\left(n^{\circ} 27\right.$ et 28-29, 2004); le «vivre

\footnotetext{
${ }^{36}$ La revue porta d'abord le titre de Qadâyâ islâmiyya, sous lequel parurent 5 numéros, de 1994 à 1998.

${ }^{37}$ Entretien avec 'Abd al-Jabbâr al-Rifâ‘'î, Qom, 28/8/03.
} 
ensemble » et le pluralisme religieux et culturel $\left(\mathrm{N}^{\circ} 31-32,2006\right)$. Ce numéro contient des plumes aussi variées que Daryush Shayegan, Mojtahed Shabestari, et un débat entre John Hick et Hossein Nasr, qui s'est tenu à Istanbul. La lecture de ses tables montre par ailleurs que la revue est perméable aux interrogations suscitées par la situation politique dans la région, notamment en Irak, où son éditeur a tenté de se réinstaller après 2003, ce qui explique l'irrégularité de sa parution.

'Abd al-Jabbâr Rifâ'î, s’il réside toujours, le plus souvent à Qom, a fondé un centre de recherche, à Bagdad: le centre d'études sur la philosophie de la religion et la nouvelle théologie, qui co-édite des livres ouvrant sur des approches scientifiques de l'islam. Ainsi d'un collectif sur l'anthropologie de l'islam ${ }^{39}$, et d'un ouvrage de 'Abd al-Jabbâr Rifâ'î, où il expose de manière très pédagogique ce qu'est la théologie moderne et les grandes questions qu'elle a traitées depuis Jamâl al-Dîn al-Afghânî ${ }^{40}$. En outre, sa revue est rééditée sous forme d'ouvrages qui constituent plusieurs collections, à Damas et à Beyrouth ${ }^{41}$. Voyons de plus près deux de ces ouvrages, qui concernent directement la nouvelle théologie. Le premier, intitulé L'ijtihâd théologique, contient plusieurs entretiens avec les Égyptiens Hasan Hanafî et Muhammad Imâra, et des Iraniens: Mojtahed Shabestari, Mostafâ Malekiân, Ahad Qarâmelki, ainsi que Sâdeq Lârijâni, réputé pour être le meilleur critique de Soroush, issu du camp conservateur, qui explique ici sa position. Le second ouvrage, Nouvelle théologie et philosophie des religions, reprend des entretiens avec certains de ces auteurs, en ajoute d'autres, ainsi que des textes et une table ronde sur la rationalité religieuse et la théologie qui regroupe des religieux et des philosophes iraniens ${ }^{42}$. On y constate, à la fois, la diversité des profils des contributeurs, et celle des approches et des opinions, tout en entrevoyant la richesse du champ intellectuel iranien.

La revue Qadâyâ islâmiyya mu'âsira a déjà beaucoup apporté à la réflexion sur la modernisation de l'islam en rassemblant, autour des mêmes questions de fond, une palette très riche de contributeurs, qui va des plus réformistes aux conservateurs, réunit des hommes de la hozeh et de l'université, des chiites d'Iran, du Liban, d'Irak et du Golfe, et des sunnites, notamment les animateurs de revues publiées à Beyrouth ou Prologues, à Casablanca. Tout ce petit monde se rencontre et converse, tant à l'occasion d'un pèlerinage à La Mecque qu'à celle d'un colloque à Rabat. Qadâyâ islâmiyya est aujourd'hui la revue plus connue dans son domaine et la mieux diffusée ; toutefois, n'est pas la seule revue éditée dans des milieux chiites qui se veulent éclairés.

\footnotetext{
${ }^{38}$ Entretien de 'Abd al-Jabbâr Rifâ‘î̀ publié dans le quotidien al-Ayyâm (Bahreïn), 26/1/2004, pris sur www.islamonline.net/Arabic/arts/2004/01/article17.shtml.

${ }_{39}$ Bâqâdir, A. B. (ed.), Anthrubûlûjiyyâ al-islâm, dâr al-Hâdî, 2005. Il contient, entre autres, des articles traduits en arabe de Jacques Waardenburg, Eric Wolf, Cliford Geertz.

${ }^{40}$ Rifầ'̂̀,'A. J, Muqaddima fî̀ al-sûu' âl al-lâhûtî al-jadîd 2005.

${ }^{41}$ À Damas, les ouvrages sont publiés à dâr al-fikr, et à Beyrouth, à dâr al-fikr al-mu'âsir et, surtout, dâr al-hâdî.

${ }^{42}$ Rifầ'î,'A. J.(éd.) 'Ilm al-kalâm al-jadîd wa falsafat al-dîn, 2002 ; al-Ijtihâd al-kalâmî..., 2002.
} 
À Damas, un disciple de Rifâ‘î publie al-Wa'î al-mu'âsir (la conscience moderne). À Beyrouth, al Muntalaq al-jadîd faite suite à al-Muntalaq et continue sur la même voie, dirigée par une femme, Zaynab Shurba. Al-Kalima (la parole) est publiée depuis le début des années 1990 par un clerc chiite saoudien, Zakî al-Mîlâd, auteur d'une douzaine d'ouvrages sur la pensée islamique contemporaine ${ }^{43}$. C'est aussi l'un de ces nouveaux réformistes «islamolibéraux ${ }^{44}$ qui, de retour en Arabie saoudite, et revenus de leurs idéaux révolutionnaires, veulent se faire entendre et dialoguer avec les composantes politiques de leur pays. Deux autres revues de ce types émanent d'institution d'enseignement. Al-Hayyât al-tayyiba est publiée par la hawza Ma'had rasûl al-akram, dont il sera question plus loin, et son rédacteur en chef en est l'ancien directeur, un clerc iranien, Najafali Mirzâ'i. La revue, créée en 1998, s'est particulièrement intéressée à la modernisation de l'enseignement religieux, pour devenir peu à peu un forum de débats éclectique, tant dans le choix des contributeurs que des thèmes traités. Ce pluralisme, que reflète la composition du comité de rédaction ${ }^{45}$, montre la marge de manœuvre dont disposent des institutions qui relèvent de l'establishment clérical iranien : la revue, comme la hawza, relèvent de l'institution qui chapeaute les écoles iraniennes à l'étranger. Il confirme aussi l'importance de l'individu au regard de l'institution: ici, le rédacteur en chef donne le ton. Al-Mahajja est une revue publiée par un institut qui dispense des cours de philosophie islamique dans la banlieue sud de Beyrouth. Elle se concentre donc sur les questions philosophiques, bien qu'elle rejoigne à maints égards les revues précédentes; on y retrouve souvent les mêmes auteurs, et parfois, les mêmes articles. La particularité d'al-Mahajja est qu'elle publie des articles de théologiens chrétiens, des auteurs libanais tel le philosophe et théologien Paul Khoury, avec lesquels l'institut, dont le directeur est aussi le rédacteur en chef de la revue, est en relation.

Enfin, plus récemment est née Nusûs mu'âsira, dont les bureaux sont à Beyrouth, mais les responsables plus souvent à Qom ou dans le Golfe. Pas étonnant puisque l'un d'eux enseigne à Qom, et que la revue est patronnée par 'Abd al-Hâdî al-Fâdilî, un clerc saoudien, ancien compagnon de Muhammad Bâqir al-Sadr à Najaf, qui s'est réinstallé en Arabie saoudite il y a quelques années. Publiée par un Centre de recherches contemporaines (Markaz al-buhûth al-mu'âsira), elle s'annonce comme «spécialisée sur la traduction de textes qui traitent de la question de la femme, de la modernité, du dialogue, de l'ouverture sur l'autre, de la philosophie du fiqh, de la théologie moderne, de la pluralité des lectures, etc. ». Pour cela, elle affirme vouloir «tirer profit de l'expérience intellectuelle contemporaine du champ culturel iranien » et se propose donc de traduire en arabe des textes qui intéressent la sphère islamique. Ces objectifs sont clairement expliqués sur la version électronique de la revue, fort

\footnotetext{
${ }^{43}$ Al-Mîlâd, Z., al-Fikr al-islâmî... 2001, p. 175.

${ }^{44}$ Cf. Lacroix, S., « Between Islamist and Liberals... », The Middle East Journal, 2004, pp. $12-13$.

${ }^{45}$ On y trouve des clercs connus comme des éclairés, tel Muhammad Hasan al-Amîn, et le «néo-conservateur» Mohammad Taqi Mesbâh Yazdi, des clercs et des intellectuels, des chiites et des sunnites (tel Ridwân al-Sayyid, animateur de la revue al-Tasâmuh).
} 
bien faite ${ }^{46}$. On y retrouve le même type de débats que ceux publiés par Qadâyâ. Ainsi, elle a par exemple produit une nouvelle traduction de Qabz va bast de Sorouch, en arguant de coupes et d'un manque de précision de la précédente, de l'importance du texte, et de la polémique qu'il a déclenchée ${ }^{47}$. En regard des options réformistes, même si l'on peut lire des analyses plus conservatrices, il s'agit bien souvent de réforme (islâh) et de renouveau (tajdîd), comme dans les autres revues. Les auteurs n'y mettent pas tous la même signification, en fonction de leurs positions, mais le but reste de trouver les modalités d'accommoder la pensée islamique au monde moderne, que ce soit, ou non, en articulation avec une vision politique de l'islam. Toutefois, il ne faut pas perdre de vue que ces revues n'intéressent qu'une élite réduite. «Certaines vendent cent cinquante exemplaires par numéro, estime Najafali Mirzâ’i, le conseiller culturel iranien, le lectorat est très limité au Liban, il est plus important au Maroc et en Égypte ${ }^{48}$. Qadâyâ islâmiyya mu'âsira est la plus lue car la plus ancienne et, certainement, la plus en prise avec les débats innovants.

\section{L'engouement pour la philosophie}

Si l'on devait tenter une distinction entre le chiisme arabe et le chiisme persan, il faudrait souligner la prédilection de ce dernier pour la mystique, la gnose ('erfân), la philosophie ; c'est d'ailleurs ce qu'avait voulu y voir, et si bien décrit Henry Corbin ${ }^{49}$. On y trouve des ordres mystiques qui sont absents dans le chiisme arabe et des théosophes qui n'y ont pas leur pareil. Si la hawza de Najaf a repris l'enseignement de la philosophie, celle-ci est devenue l'une des spécificités de Qom dans laquelle se retrouvent, paradoxalement, les camps opposés des conservateurs partisans du régime et des réformistes, même s'ils ne l'appréhendent pas de la même manière. Pour les premiers, gnose et philosophie servent une vision du monde qui promeut un islam total, une utopie islamique, alors que les seconds en font les vecteurs de l'individuation de la religion.

Au Liban, une génération de clercs formés à Qom après la révolution est revenue imprégnée d'un savoir que n'ont pas leurs aînés issus du moule de Najaf, dans les années 1960. Ils le transmettent à leur tour. Ainsi, en 1999, le cheikh Shafî̀ Jarâdeh a fondé l'Institut de théosophie pour les études religieuses et philosophiques (Ma'had al-ma'ârif al-hikmiyya li1-dirâsât al-dîniyya wa al-falsafiyya) dans la banlieue sud de Beyrouth. L'enseignement, dispensé le soir, s'organise en deux sections : l'une pour les étudiants inscrits, qui doivent réussir un certain nombre de cours de philosophie, 'irfân, croyances fondamentales ('aqîda), théologie, morale, etc.) pour réussir un examen (non reconnu par l'État); l'autre, ouverte à tous, propose des cours de méthode, de soufisme et de 'irfân. L'objectif est moins de former des spécialistes que «d'élargir l'horizon culturel» des étudiants de la hawza ou de

\footnotetext{
${ }^{46}$ www.nosos.net. On consultera notamment, «Li-mâdhâ nusûs mu'âsira » (Pourquoi Nusûs mu'âsira), par Haydar Hubb Allâh, rédacteur en chef de la revue.

${ }^{47}$ Cf. Nusûs mu'âsira, n ${ }^{\circ}$, hiver 2006, p. 136.

${ }^{48}$ Entretien au centre culturel iranien, Beyrouth (Bîr Hasan), 21/12/06.
} 
l'université sur ces disciplines et de guider leurs recherches en leur inculquant une méthode. Les droits d'inscription, relativement peu élevés, visent surtout à trier les étudiants sérieux car l'institut est soutenu financièrement par «le khums de la marja 'iyya », c'est-à-dire Ali Khamenei. Shafîq Jarâdeh a commencé par enseigner un ouvrage classique de 'irfân " ${ }^{50}$, ainsi que des ouvrages de 'Allâma Tabâtabâ'i, considéré comme le fondateur du cercle philosophique de Qom ${ }^{51}$, puis il a élargi ses centres d'intérêt, notamment à l'histoire de la philosophie et la théologie chrétienne. «John Hick a influencé la pensée iranienne contemporaine, et je pense qu'il faut le lire, comme d'autres théologiens chrétiens, pour tirer profit de leur expérience », déclare-t-il. Bien plus, un cercle de débats réunit régulièrement musulmans et chrétiens où chacun fait part de ses positions sur les questions communes qui se posent aux religions. L'institut entretient ainsi un dialogue avec Paul Khoury, «qui joue le rôle de John Hick au Liban », l'évêque Georges Khodr ou le père Mouchir Aoun, à l'université de Kaslik ${ }^{52}$. «Si nous ne débattons pas des questions qui nous intéressent dans le cadre de la religion, plus large que celui de l'islam, nous ne nous développerons pas », estime Shafî̀ Jarâdeh. «Et si Dieu est pour tous, comment puis-je le monopoliser ? Il faut dialoguer, envisager les points sur lesquels nous nous accordons et ceux sur lesquels nous divergeons ${ }^{53}$. Ce discours trouve des auditeurs dans la banlieue sud de Beyrouth, où les jeunes sont en demande d'une autre approche de l'islam chiite que celle du culte quotidien et de ses règles, voire en quête de spiritualité. Philosophie et 'irfân attirent en outre les étudiants militants ou sympathisants du Hezbollah: ils y puisent un contenu intellectuel qui soutient leur engagement. Shafîq Jarâdeh est d'ailleurs réputé pour être proche du parti. Les femmes sont aussi présentes à l'institut, et elles sont même majoritaires autour de Muhammad al-Hâjj, un clerc qui dirige un autre institut de la banlieue sud de Beyrouth, l'Institut de la philosophie orientale (Ma'had al-ishrâq), sorte de centre culturel où l'on vient, le soir, se plonger dans la métaphysique et la théosophie.

La philosophie transcendantale de Mollâ Sadrâ Shirâzi a aussi été introduite dans la hawza au Liban, tout comme des disciplines nouvellement constituées qui visent à moderniser l'enseignement des sciences religieuses. Certes, les écoles concernées sont, en fait, des instituts réformés qui ne fonctionnent pas selon le système de la hawza classique, « libre », où chaque étudiant peut choisir ses maîtres et ses cours, mais selon le modèle universitaire ${ }^{54}$. Au Liban, les plus modernistes d'entre eux sont dirigés par de jeunes clercs et placés l'un sous

\footnotetext{
${ }^{49}$ Corbin, H., En islam iranien, 1971.

${ }^{50}$ Mahdî Bahr al-'Ulum, al-Sayr wa al-sulûk. Il s'agit d'un traité de gnose pratique, celle-ci décrivant des méthodes pour adorer Dieu (dhikr, contemplation, etc.).

${ }^{51}$ Cf. Rifâ‘î, 'A. J., Tatawwur al-dars al-falsafî, 2005, chap. IV.

${ }^{52}$ Paul Khoury, est théologien et philosophe à l'Institut Saint-Paul de Philosophie et de Théologie, à Harissa. Georges Khodr, évêque de l'Eglise grecque orthodoxe du Mont-Liban, a beaucoup œuvré pour le renouveau de L'Église. Mouchir Aoun enseigne la philosophie et le dialogue interculturel à la faculté de théologie de l'université de Kaslik.

${ }_{53}^{53}$ Entretien avec Shafîq Jirâdeh, Hârat Hurayk, 16/05/06.

${ }^{54}$ Sur la réforme de la hawza, Mervin, Sabrina, « La hawza à l'épreuve du siècle... », 2003.
} 
l'autorité religieuse de Muhammad Husayn Fadlallâh, et l'autre sous celle de Ali Khamenei ${ }^{55}$. En plus des sciences humaines, profanes (sociologie, psychologie, pédagogie), de nouvelles disciplines des sciences religieuses islamiques y ont été introduites. S'il est désormais admis d'enseigner une approche comparée du droit islamique, entre écoles chiites et écoles sunnites, il est plus nouveau de le mettre, dans un autre cours, en rapport avec le droit positif. Globalement, le fiqh se ramifie : on étudie son histoire, ainsi que le fiqh pratique, social ; les normes (ahkâm), les fatwâ, etc. La philosophie du fiqh, discipline apparue d'abord dans les revues, a fait son entrée dans les programmes, tout comme la nouvelle théologie. Les débats entre oulémas et intellectuels religieux trouvent, à terme, une application pratique dans la mise en place de nouveaux programmes.

L'institut al-Rasûl al-akram et sa branche féminine, al-Zahrâ', sont considérés, dans la sphère islamique comme des écoles religieuses avant-gardistes. La lecture de son corpulent «catalogue » 2003-2004 ${ }^{56}$ qui présente les cours, année par année, révèle un souci de catégorisation nouvelle des disciplines, une volonté d'organisation de l'enseignement et une ouverture sur la littérature des sciences humaines produites à l'étranger, le tout servi par une maquette claire et élégante. Le directeur, très au fait des questionnements qui parcourent les milieux religieux, affiche son objectif en introduction : moderniser la hawza sans lui enlever sa profondeur ${ }^{57}$. On serait tenter d'y déceler l'expérience iranienne en matière de fondation et d'administration d'établissements de ce type, puisque celui-ci suit la marja'iyya de Ali Khamenei. En fait, il faut aussi y voir la marque d'initiatives individuelles - comme toujours dans les institutions chiites, qui ont des difficultés à se pérenniser en tant que telles plutôt qu'en référence à un fondateur. L'institut a été dirigé par Najafali Mirzâ'i, un clerc très en prise avec les débats actuels, rédacteur en chef d'une revue qui les publicise, al-Hayât altayyiba, ${ }^{58}$. Il a, depuis, fondé à Beyrouth un centre d'études visant à faire connaître les civilisations arabe et iranienne l'une à l'autre (Markaz al-hadâra li-l-dirâsât al-îrâniyya al'arabiyya), et surtout leurs courants de pensée actuels ${ }^{59}$.

\section{L'empreinte de l'Iran?}

Lorsqu'on lui demande si ce goût pour la philosophie et la nouvelle théologie est un effet de l'influence iranienne, Najafali Mirzâ'i estime que non. «Les gens étudient Mollâ Sadrâ Shirâzi parce qu'il est chiite, et pas parce qu'il est iranien ». Il va même plus loin : «Les grands penseurs sont universels. Ibn 'Arabî, par exemple, n'est pas « sunnite arabe », il

\footnotetext{
${ }^{55}$ Il s'agit respectivement d'al-Ma‘had al-shar'î al-islâmî, et de Ma‘had al-rasûl al-akram, tous deux situés dans la banlieue sud de Beyrouth. Ibid., p. 77 sq.

${ }_{57}^{56}$ Dalîl Ma'had al-rasûl al-akram, 2003-2004, 471 p.

${ }^{57}$ Ibid., p. 10-11.

${ }^{58}$ Entre temps, celui-ci a fondé un centre de recherche à Qom, puis est revenu au Liban en tant que conseiller culturel.

${ }^{59}$ Le centre traduit des ouvrages dans les deux langues sur des sujets divers, allant de la poésie contemporaine à la philosophie du fiqh, le droit de la femme, les fondements théoriques de la République islamique. Entretien à Bir Hasan, Beyrouth, 21/12/06.
} 
appartient à tous ${ }^{60}$. De même, aujourd'hui, Soroush ou Shayegan, ne sont pas des penseurs iraniens... ». À la même question, Muhammad al-Hâjj répondait, non sans humour : « Notre association ne suit pas l'Iran, elle suit les idées de Shirâz. Ici, on ne s'intéresse pas à l'idéologie, mais à la métaphysique ${ }^{61}$. Shafîq Jarâdeh, lui, déclare que l'histoire de la philosophie n'étant pas enseignée, il a essayé de le faire « à l'iranienne », tout en ajoutant des auteurs arabes.

Quant à la «nouvelle théologie », elle n'est pas si nouvelle, si l'on en croit certains. Ses détracteurs considèrent, sans doute avec quelque mauvaise foi, que l'on a revêtu d'habits neufs une ancienne discipline, autrement dit que seules les formulations ont changé. Ses partisans, eux, en font l'histoire. Ainsi, 'Abd al-Jabbâr Rifâ‘î remonte au savant indien Shibli Nu'mâni (m. 1914), auteur d'un ouvrage intitulé La nouvelle théologie. Puis il mentionne le fameux réformiste égyptien Muhammad 'Abduh (m. 1905) et son traité de l'unicité (Risâlat al-tawhîd) et, enfin, Mohamamd Iqbâl (m. 1938) qui, plus que les précédents, nourrit véritablement le projet de reconstruire les sciences religieuses en islam ${ }^{62}$. L'étape suivante est constituée par les écrits de Muhammad Hosein Tabâtabâ'i, de son disciple Mortaza Motahhari, et de Muhammad Bâqir al-Sadr ${ }^{63}$. Ensuite, selon lui, la force de l'Iran fut de produire des intellectuels dotés à la fois d'une formation classique de hozeh et d'une formation scientifique, académique, moderne ${ }^{64}$. Ainsi, ils ont pu penser l'islam avec les outils de leur temps. Bon nombre de partisans de la nouvelle théologie font un parallèle entre l'ouverture des penseurs d'aujourd'hui sur la philosophie et les sciences sociales occidentales et le mouvement de traduction de la philosophie grecque au IXe siècle qui a permis le développement de la falsafa. Sauf que, objecte Mohammad Mojtahed Shabestari « au temps de Ma'mûn, les musulmans dominaient le monde et traduisaient, alors que maintenant, ils empruntent des idées qui se veulent universelles ».

Si cette théologie islamique moderne résulte d'un processus historique, elle n'est pas réservée à l'Iran. Certes, l'existence de la République islamique a soulevé des questions qui ont favorisé son développement, mais selon Shabestari lui-même, elle est pratiquée ailleurs, dans le monde musulman. Ainsi, selon lui, les écrits de l'égyptien Nasr Abu Zayd ou du syrien Muhammad Shahrûr, même s'ils s'inscrivent dans l'exégèse coranique, relèvent aussi de la nouvelle théologie, puisqu'ils promeuvent une nouvelle vision de l'islam. En outre, il considère Mohammad Arkoun et Mohammad Abed al-Jabiri comme des théologiens modernes ${ }^{65}$.

\footnotetext{
${ }^{60}$ Précisons qu'Ibn 'Arabî a une place considérable dans le 'irfân théorique chiite car ses théories ont été adoptées par Mollâ Sadrâ. D’où la pertinence de cet exemple.

${ }^{61}$ Entretien dans la banlieue de Beyrouth, janvier 2002.

${ }^{62} \mathrm{Cf}$. Iqbal, Mohammed, Reconstruire la pensée religieuse de l'islam, 1996.

${ }^{63}$ Cf. l'introduction de A. J. Rifâ‘î à Shabestari, M. M., Madkhal ilâ 'ilm al-kalâm al-jadîd, 2000 , p. 7-9. Voir aussi la comparaison qu'il établit entre 'Abduh et Iqbâl dans « Muhammad 'Abduh wa Muhammad Iqbâl, ru’yatân fî̀ tahdîth al-tafkîr al-dînî », 2006, pp. 99-114.

${ }^{64}$ Entretien à Qom, 28/8/03.

${ }^{65}$ Entretien à Téhéran, 16/8/03.
} 
C'est bien l'avis de Hasan Jâbir, qui mentionne les mêmes noms, auxquels il ajoute celui du libanais 'Alî al-Harb. L'historicité du texte sacré a été étudiée par les auteurs arabes, avant Soroush : «aussi, sa lecture fut un choc pour les Iraniens, pas pour nous », déclare-til ${ }^{66}$. Si, quelques années auparavant, on pouvait entendre, dans la «sphère islamique », des réflexions telles que : « nous faisons comme à Qom », ou bien : « cela vient d'Iran », ce n'est plus le cas aujourd'hui. Plus qu'une raison politique, il faut y voir un effet d'habitude et une expression de l'attachement à la culture arabe. En outre, les penseurs réformistes, qui se veulent ouverts sur le monde, le sont aussi sur les mondes musulmans et veulent dépasser les appartenances identitaires pour ne prendre en compte que les affinités intellectuelles. Enfin, n'oublions pas les figures de la scène religieuse et intellectuelle locale qui ne se réfèrent pas à l'Iran et ne se disent pas influencés par ses penseurs, même s'ils les lisent. Ainsi de Muhammad Husayn Fadlallâh, qui poursuit son chemin en tant que marja' et applique, dans ce cadre, un réformisme plus pratique que théorique, ou de Muhammad Hasan al-Amîn, ancré dans sa société et dans le chiisme local, arabe ${ }^{67}$.

\section{Bibliographie}

\section{A. Jabar, Faleh,}

B. The Shi'ite movement in Iraq, Saqi, Londres, 2003.

Al-Amîn, Muhammad Hasan,

al-Ijtimâ' al-'arabî al-islâmî. Murâja' ât fî al-ta'addudiyya wa al-nahda wa al-tanwîr [La société arabe islamique. Études sur le pluralisme, l'essor et les Lumières], dâr al-Hâdî, Beyrouth, 2003.

Amirpur, Katajun

« Mohammad Mojtahed Shabestari », Orient, 41/1 (2000), pp. 14-17.

Bâqâdir, Abû Bakr Ahmad (ed.), Anthrubûlûjiyâ al-islâm [Anthropologie de l'islam], dâr alHâdî, Beyrouth, 2005.

Bonaud, Yahya Christian

L'Imam Khomeiny, un gnostique méconnu du XXe siècle : Métaphysique et théologie dans les œuvres philosophiques et spirituelles de l'Imam Khomeiny, Beyrouth, al-Bouraq, 1997.

Buchta, W., Who rules Iran? The Structure of Power in the Islamic Republic, Washington Institute for Near East Policy-Konrad Adenauer Stiftung, Washington, 2000.

Beheshti, Muhammad al-Iqtisâd al-islâmî, (traduction en arabe) [L'économie islamique], Râbitat al-thaqâfa wa al'alâqât al-islâmiyya, Téhéran, 1997.

Chehabi, Houchang E. (ed.)

\footnotetext{
${ }^{66}$ Entretien à Beyrouth, 3/1/07.

${ }^{67}$ Sur M. H. Fadlallâh, voir son site : www.bayynat.org ; pour M. H. al-Amîn, son ouvrage, al-Ijtimâ' al-'arabî al-islâmî, 2003.
} 
Distant relations. Iran and Lebanon in the last 500 years, Centre for Lebanese Studies, I. B. Tauris, Londres, 2006,

COLE, Juan

Sacred space and Holy War. The Politics, Culture and History of Shi'ite Islam, I.B. Tauris, 2002.

Collectif de oulémas

Sîra wa hayât al-shahîd Bihishtî [Biographie du martyr Beheshti], Dâr al-Hâdî, Beyrouth, 2000.

Corbin, Henry

En islam iranien aspects spirituels et philosophiques, Gallimard, Paris, 1971-1972.

Dabashi, Hamid

Theology of Discontent, New York University Press, 1993.

Digard, Jean-Pierre, Hourcade, Bernard et Richard, Yann

L'Iran au XXe siècle, Fayard, Paris, 1996.

DONOHUE, J.

« Notre économie », Cahiers de l’Orient, n8-9, 1988, pp. 179-202.

Farhat, Nabil

«Histoire d'une inimitié fraternelle. Le Hezbollah contre Fadlallah», Hebdo Magazine, 7/3/2003, pp. 8-14.

FISCHER, Michael M.J.

Iran from Religious Dispute to Revolution, Harvard University Press, Cambridge-Londres, 1980.

Hairi, Abdul-Hadi

Shi'ism and Constitutionalism in Iran. A Study in the Role Played by the Persian Residents of Iraq in Iranian Politics, E.J. Brill, Leiden, 1977.

Iqbal, Mohammed

Reconstruire la pensée religieuse de l'islam, trad de l'anglais par Eva de Vitray-Meyerovitch, ed. du Rocher/ed UNESCO, 1996.

Jâbir, Hasan

Al-Maqâsid al-kulliyya wa al-ijtihâd al-mu'âsir [Les maqâsid et l'ijtihâd moderne], Dâr alhiwâr, Beyrouth, 2001.

«al-Tajdîd wa al-taqlîd fî̀ al-fikr al-îrânî al-mu'âsir » [Renouveau et tradition dans la pensée iranienne contemporaine], Shu'ûn al-sharq al-awsat, 103, Beyrouth, été 2001, p. 38-47.

Kadîvar, Muhsin,

Nazariyyât al-dawla fì al-fiqh al-shî‘ î [Les théories de l'État dans le droit islamique chiite], Dâr al-Hâdî, Beyrouth, 2004.

Khoskhoravar, Farhad

« Neo-conservative Intellectuals in Iran », Critique, n 19 , Fall 2001, p. 5-30.

Lacroix, Stéphane

«Between Islamist and Liberals : Saudi Arabia's new "Islamo-Liberal" Reformists », The Middle East Journal, 58/3, été 2004, p. 345-365.

Luizard, Pierre-Jean 
La vie de l'ayatollah Mahdî al-Khâlisî par son fils, introduction, annotation et traduction Pierre-Jean Luizard, La Martinière, Paris, 2005.

Mallat, Chibli

The renewal of Islamic law. Muhammad Baqer as-Sadr, Najaf and the Shi' $i$ International, Cambridge University Press, Grande Bretagne, 1993.

MARTIN, Vanessa

Islam and Modernism : the Iranian Revolution of 1906, I.B. Tauris, Londres, 1989.

Creating an Islamic State. Khomeini and the Making of a New Iran, I.B. Tauris, London, New York, 2003.

Merat, Zarir

«Les revues intellectuelles. L'embryon d'une agora », Les cahiers de l'Orient, 1er trimestre $1998, n^{\circ} 49$, pp. 87-102.

Mervin, Sabrina

«La hawza à l'épreuve du siècle : la réforme de l'enseignement religieux supérieur chiite de 1909 à nos jours », Maher al-Charif et Salam al-Kawakibi éd., Le courant réformiste musulman et sa réception dans les sociétés arabes, IFPO, Damas, 2003, pp. 69-84.

Mesbâh Yazdî, al-'allâma al-shaykh

Usûl al-ma'ârif al-insâniyya [Les fondements des connaissances humaines], Mu'assasat Umm al-qurâ, Beyrouth, 2004.

Al-Mîlâd, Zakî

Al-Fikr al-islâmî, tatawwurâtuhu wa masâratuhu al-fikriyya, dâr al-hâdî, Beyrouth, 2001.

NAEF, Silvia

"Shî‘̂े-shuyû‘'î or: How to become a Communist in a Holy City », The Twelver Shia in Modern Times. Religious Culture and Political History, Werner Ende et Rainer Brunner éd., Leiden, Brill, 2001, p. 255-267.

NIKPEY, Amir

Politique et religion en Iran contemporain. Naissance d'une institution, Paris, l'Harmattan, 2001.

Rahnema, Ali

An Islamic Utopian. A political Biography of Ali Shari 'ati, I. B. Tauris, Londres, 2000.

Richard, Yann, «Le rôle du clergé : tendances contradictoires du chi'isme iranien contemporain», Archives des sciences sociales des religions, 55/1, 1983, p. 5-27.

L'Iran. Naissance d'une république islamique, La Martinière, Paris, 2006.

Ridgeon, Lloyd

Religion and Politics in Modern Iran. A Reader, I. B. Tauris, Londres, 2005.

Rifâ‘̂̂,'Abd al-Jabbâr ed.

'Ilm al-kalâm al-jadîd wa falsafat al-dîn [La théologie moderne et la philosophie de la religion], dâr al-Hâdî, Beyrouth, 2002.

Al-Ijtihâd al-kalâmî. Manâhij wa ru'â mutanawwi'a fî al-kalâm al-jadîd [L'ijtihâd théologique. Méthodes et visions diverses sur la nouvelle théologie], dâr al-Hâdî, Beyrouth, 2002.

Rifâ'î,'Abd al-Jabbâr 
«Muhammad 'Abduh wa Muhammad Iqbâl, ru'yatân fî̀ tahdîth al-tafkîr al-dînî » [Muhammad 'Abduh et Muhammad Iqbâl, deux visions de la modernisation de la pensée religieuse], Hadâthât islâmiyya/Modernités islamiques, ed. Charif, Maher et Mervin, Sabrina, IFPO, Damas, 2006, pp. 99-114.

Rifâ'‘̂,'AAbd al-Jabbâr

Muqaddima fî al-sû̀'âl al-lâhûtî al-jadîd [Introduction à la question théologique moderne], dâr al-hâdî, Beyrouth, 2005.

Tatawwur al-dars al-falsafí fî al-hawza al-'ilmiyya [Le développement de l'enseignement de la philosophie dans la hawza], Dâr al-Hâdî, Qadâyâ islâmiyya mu'âsira, Beyrouth, 2005.

Rosiny, Stephan,

"The Tragedy of Fâtima al-Zahrâ' in the Debate of two Shiite Theologians in Lebanon », in The Twelver Shia in Modern Times, ed. Rainer Brunner et Werner Ende, Brill, 2001, pp. $207-$ 219.

AS-SADR, Muhamamd Bâqir,

Lessons in Islamic Jurisprudence, trad. Roy Mottahedeh, Oneworld Publications, Oxford, 2003.

Sadri, Mahmood

«Sacral Defense of Secularism : The Political Theologies of Soroush, Shabestari and

Kadivar », International Journal of Politics, Culture and Society, 15/2, hiver 2001, pp. 257-

270.

Sankari, Jamal,

Fadlallah. The Making of a Radical Shi 'ite Leader, Saqi Books, 2005.

Shabestari, Mohammad Mojtahed,

Madkhal ilâ 'ilm al-kalâm al-jadîd, Dâr al-Hâdî, Beyrouth, 2000.

Vahdat, Farzin

« Post-revolutionary Discourse of Mohammad Mojtahed Shabestari and Mohsen Kadivar :

Reconciling the Terms of Mediated Subjectivity. Part I : Mojtahed Shabestari », Critique, 16

(printemps 2000), p. 31-54 ; « Part II: Mohsen Kadivar », Critique, 17 (2000), p. 135-157.

Walbridge, Linda (ed.)

The most Learned of the Shi'a. The Institution of the Marja' Taqlid, Oxford University Press, 2001.

Wâsifî, Muh Ridâ

al-Fikr al-islâmî al-mu'âsir fî̀ Irân. Jadaliyyat al-taqlîd wa al-tajdîd [La pensée islamique contemporaine en Iran. Problématique de la tradition et du renouveau], Dâr al-jadîd, Beyrouth, 2001.

Dalîl Ma 'had al-rasûl al-akram al- 'âlî li-l-sharî'a wa al-dirâsât al-islâmiyya, Beyrouth, 2003-2004, 
